\title{
Extreme Value Charts \& Analysis of Means Based on the New Weighted Exponential Distribution
}

\author{
Anil Arepalli 1.*, B. Srinivasa Rao ${ }^{2}$ \\ ${ }^{1}$ Assistant Prof., Faculty of Statistics, Department of Basic Science \& Humanities, \\ Dhankeula Institute of Engineering \& Technology. anilarepalli08@gmail.com \\ ${ }^{2}$ Associate Prof., Faculty of Statistics, Department of Mathematics \& Humanities, \\ R.V.R \& J.C College of Engineering. \\ ${ }^{*}$ Corresponding author: anilarepalli08@gmail.com
}

\begin{abstract}
It is assumed that the probabilistic model of the quality characteristics follows the new weighted exponential distribution. Control charts based on each subgroup's extreme values are established. The constants in the control chart are determined by the probability distribution of the extreme value order statistics of the sub-group and the sub-group size. The proposed chart is thus referred to as an extreme values chart. A biased overall mean analysis method (ANOM for truncated population) is used for the new weighted exponential distribution. Examples based on real time data are used to explain the findings.
\end{abstract}

Keywords: ANOM, Equi-tailed, In-control, NWED, Q-Q plot 
1. Introduction

Consider New Weighted Exponential Distribution (NWED) with probability density function (PDF) as follows;

$f(x ; \alpha, \lambda)=(1+\lambda) \alpha \cdot e^{-(1+\lambda) \alpha x} ; \quad x>0, \alpha>0, \lambda>0$

Its cumulative distribution function ( $c d f$ ) is given by,

$F(x ; \alpha, \lambda)=1-. e^{-(1+\lambda) \alpha x} ; \quad x>0, \alpha>0, \lambda>0$

Where, $\alpha$ is a scale parameter and $\lambda$ is a shape parameter.

The extreme order statistical percentiles of the NWED sample are required to create a control chart that uses extreme observations from a subset of manufacturing processes with NWED quality options. Specifically, the first test vector $X=\left(x_{1}, x_{2}, \ldots, x_{n}\right)$ from the continuous processing is used as the test statistic on the extreme value control chart. The control chart in extreme value chart displays entire sample observations, but no statistic(s) is/are calculated from it. According to one or both extreme values of the sample, $x_{1}$ (test least) and $x_{n}$ (test most extreme), fall below or above two defined lines (limits), a corrective action is taken. Therefore, this chart is called an extreme value control chart [9].

Many professionals use Shewart control charts as a statistical method [5] for quality control. If the solution is found, the technique shall be adjusted when such charts indicate that an assignable cause exists [1]. In the abstract group statistical for which the control chart is built, the existence of an assignable cause is understood as a signal of heterogeneity $[1,8]$. For example, the mean process would be heterogeneous when the figures are sample mean, which would signify differences from the goal mean [4]. Such an analysis is often done by means of means to split a collection of different subset mean into categories [2], so that means are homogenous within a category and heterogeneous between categories and the technique is known as an analysis of means (ANOM) as described by Ott.E.R [7]. The control chart for the mean is read differently using the ANOM technique $[6,10]$ : grouping of the plotted means within or beyond the control limits. The two means must fall under the control limits in order for all of them to be homogeneous. The probability of any sub-group is equal to the coefficient of confidence, take is as $(1-\alpha)$. This probability statement will be the $n^{\text {th }}$ power of the likelihood that the mean of a subgroup fall within the boundaries, provided it is supposed to be independent. I.e. the confidence interval of $\bar{x}$ for the distribution of samples should be equivalent to $(1-\alpha)^{1 / n}$ between two specified bounds. In the rest of this article, the same principle is also applied by NWED. We only looked at ANOM control charts [3] in this research since it intends to examine ANOM by employing extreme value statistical control limits. No new ANOM tables or procedures have been examined by us. However, there is a thorough documentary on ANOM by Rao.C.V [9] and certain similar works are in this direction [11-14] are mentioned in references.

The rest of the paper is described below. Section 2 gives a fundamental exposure to extreme value control diagrams that are supported by average runtime (ARL) and to ANOM. In Section 3, NWED is used with an ANOM in conjunction with numerical examples employing extreme value control charts of NWED. The findings and conclusions of Section 4 are provided.

\section{Materials and methods}

The mathematical and statistical research background of Extreme value charts \& ANOM and the methods for the study of NWED model are discussed in this part.

\subsection{Extreme Value Chart for NWED Model}

NWED model is considered to be followed by the sample observations. The theory of extreme order statistics, based on the NWED model, determines the control lines. The 
control lines should be chosen such that an arbitrarily chosen $x_{i}$ of $X=\left(x_{1}, x_{2}, \ldots, x_{n}\right)$ lies inside the limits with probability $(1-\alpha)^{1 / n}$. The following formula can be used to express this as a probability inequality:

$P\left(x_{1} \leq L\right)=\alpha / 2 \& P\left(x_{n} \leq U\right)=\alpha / 2$. The cumulative distribution functions of the lowest and largest order statistics in a sample of size $n$ from any continuous population are $[F(x)]^{n}$ along with $1-[1-F(x)]^{n}$ commonly, according to the theory of order statistics, here $F(x)$ is the population's distribution function. The value of $\alpha$ will be 0.0027 , if $1-\alpha$ were needed at 0.9973 . Using $F(x)$ as the CDF of a NWED model, we can find solutions to the two equations $1-[1-F(x)]^{n}=0.00135$ and $[F(x)]^{n}=0.99865$, which can then be used to establish the extreme value chart's control limits.

\subsubsection{Average Run Length (ARL) of the Extreme Value Chart}

For a given parametric combination $\alpha$ and $\lambda$ of NWED and a given sample size $n$ and if $L \& U$ respectively stand for the lower control limit $(L C L)$ and upper control $\operatorname{limit}(U C L)$ of the extreme value control chart, then the ARL of that control chart is given by, $A R L=\frac{1-p}{p}$, where $p=2-[F(U)]^{n}-[1-F(L)]^{n}$. Here $F(x)$ denotes the distribution function of NWED with parameters $\alpha$ and $\lambda$. We have to substitute $L \& U$ to get the value of $\mathrm{p}$ and hence to get ARL-average run length. Generally, ARL decreases as $n$ increases.

\subsection{Analysis of Means (ANOM) for NWED Model}

Assume that $\overline{x_{1}}, \overline{x_{2}}, \ldots, \overline{x_{k}}$, are arithmetical means of a NWED model in $k$ subgroups of the size $n$. When control charts are established by employing these sub-group means, whether acceptable qualitative changes exist can be assessed in the original population from which these sub-groups come to be drawn. Depending on the elementary population distribution, one can adopt the constants of control diagram we created or the usual Shewart constants from statistical textbook. Broadly speaking, when all means in the subsets are inside the control limits, the mechanism is considered to be beneath control. Otherwise, the mechanism would be excluded from control. If $\alpha$ is the amount of significance of the above decisions, the following likely claims can be made.

$P\left(L C L<\overline{x_{i}}<U C L\right)=1-\alpha, \quad \forall i=1,2, \ldots, \mathrm{k}$

Using the notion of independent subgroups Eq. (3) becomes

$$
P\left(L C L<\overline{x_{i}}<U C L\right)=(1-\alpha)^{1 / k}
$$

With Equi-tailed probability for each subgroup mean, we can find two constants say $L^{*}$ and $U^{*}$ such that

$P\left(\overline{x_{i}}<L^{*}\right)=P\left(\overline{x_{i}}>U^{*}\right)=\frac{1-(1-\alpha)^{1 / k}}{2}$

$L^{*}$ and $U^{*}$ satisfy $U^{*}=-L^{*}$, when the population drawn from a Normal distribution. We must measure $L^{*} \& U^{*}$ separately from sampling distribution of $\bar{x}_{i}$ for skewed populations like NWED. As a consequence, these are dependent on the number of subgroups $k$ and sub-groups size $n$. Percentiles of the sampling distribution for $\bar{x}$ in NWED model were calculated using simulation process (Monte-Carlo) and are shown in Table 5, Table 6 and Table 7. For stated $n$ and $k$, we use the percentiles in Eq. (5) to get $L^{*}$ and $U^{*}$ for $\alpha=0.0 .1,0.05$, and 0.10 . Table 5, Table 6 and Table 7 contain this detail. 


\section{Results and discussion}

The solutions to the two equations $1-[1-F(x)]^{n}=0.00135$ and $[F(x)]^{n}=0.99865$ for $n=2,3, \ldots, 10$ are denoted as $Z_{(1) 0.00135} \& Z_{(n) 0.99865}$ are listed in Table 1.

Table 1. Control Limits of Extreme value charts.

\begin{tabular}{cccccccccc}
\hline$n$ & 2 & 3 & 4 & 5 & 6 & 7 & 8 & 9 & 10 \\
\hline$Z_{(1) 0.00135}$ & 0.0021 & 0.0014 & 0.001 & 0.0008 & 0.0007 & 0.0006 & 0.0005 & 0.0004 & 0.0004 \\
$Z_{(n) 0.99865}$ & 22.8139 & 24.0806 & 24.9794 & 25.6767 & 26.2463 & 26.728 & 27.1453 & 27.5133 & 27.8425 \\
\hline
\end{tabular}

The values from Table 1 express the subsequent probability statement;

$P\left(Z_{(1) 0.00135}<Z_{i}<Z_{(n) 0.99865}\right)=0.9973, \quad \forall i=1,2, \ldots, n$

$P\left(\sigma Z_{(1) 0.00135}<x_{i}<\sigma Z_{(n) 0.99865}\right)=0.9973, \quad \forall i=1,2, \ldots, n$

Taking $\bar{x} / 3.125$ as an unbiased estimate of $\sigma$ for a specific parametric combination $\alpha=0.2$ and $\lambda=0.6$ of NWED by the simulation process, the above equation becomes

$P\left(L \bar{x}<x_{i}<U \bar{x}\right)=0.9973, \quad \forall i=1,2, \ldots, n$

Where $L=\frac{Z_{(1)(0.00135)}}{3.125}$ and $U=\frac{Z_{(n)(0.99865)}}{3.125}$. Thus $L \& U$ would establish the control chart constants for extreme value charts. Table 2 display these values for $n=2(1) 10$.

Table 2. Constants of Extreme value charts.

\begin{tabular}{cccccccccc}
\hline$n$ & 2 & 3 & 4 & 5 & 6 & 7 & 8 & 9 & 10 \\
\hline$L$ & 0.0006 & 0.0004 & 0.0003 & 0.0002 & 0.0002 & 0.0001 & 0.0001 & 0.0001 & 0.0001 \\
$U$ & 7.3004 & 7.7058 & 7.9934 & 8.2165 & 8.3988 & 8.5529 & 8.6865 & 8.8042 & 8.9096 \\
\hline
\end{tabular}
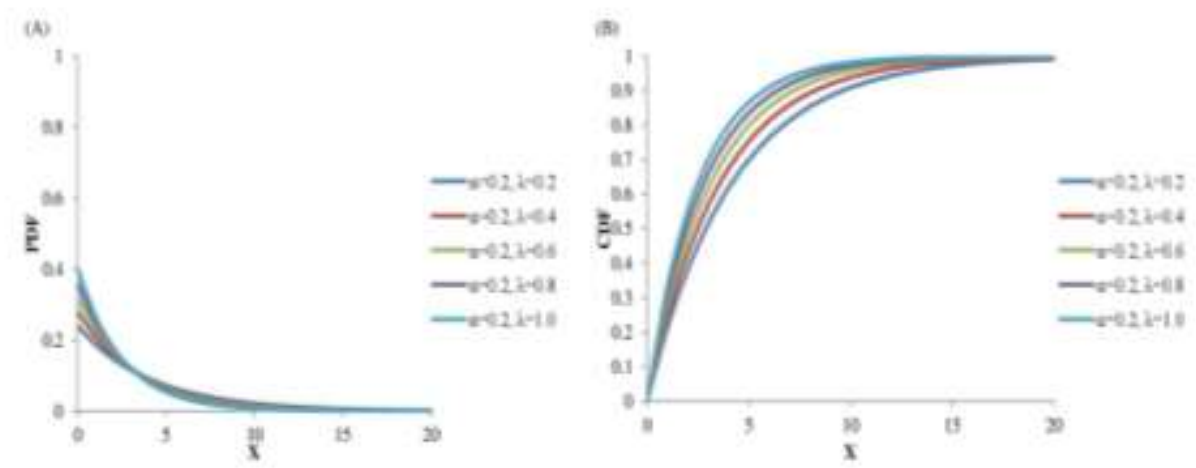

Figure 1 Plots of the $p d f$ 's and $c d f$ 's of NWED for selected parameter values.

(A): Probability Density Function, (B): Cumulative Distribution Function.

For a fixed parametric combination $\alpha=0.2$ and $\lambda=0.6$ of NWED and a given sample size $n$ and if $L \& U$ respectively stand for the lower control limit $(L C L)$ and upper control $\operatorname{limit}(U C L)$ of the extreme value control chart, then the ARL of that control chart is given by, $A R L=\frac{1-p}{p}$, where $p=2-[F(U)]^{n}-[1-F(L)]^{n}$. Here $F(x)$ denotes the distribution function of NWED with parameters $\alpha=0.2$ and $\lambda=0.6$. We have to substitute $L \& U$ to get the value of $\mathrm{p}$ and hence to get ARL-average run length. These values are shown in Table 3 for $\mathrm{n}=2$ to 10 . 
Table 3. Average Run Length (ARL)

\begin{tabular}{cccccccccc}
\hline$n$ & 2 & 3 & 4 & 5 & 6 & 7 & 8 & 9 & 10 \\
\hline ARL & 4.4207 & 3.2697 & 2.6218 & 2.1982 & 1.8967 & 1.6694 & 1.4909 & 1.3467 & 1.2274 \\
\hline
\end{tabular}

An In-Control result of a mean control chart shows that, even if means of sub-group differ significantly, they are all homogeneous in some way. In a study of analysis of variance, this is the null hypothesis. As a consequence, the control limits can be used instead of the study of analysis of variance. The Ott.E.R, [7] tables can be used with a normal population. The constants in Table 5, Table 6 and Table 7 can be used for a NWED. For research purpose, we've included some examples below in which the Q-Q plot technique (graphical tool to assess linearity between actual data and theoretical model) was used to determine the goodness of fit of the NWED model and the homogeneity of means was evaluated in each case.

\subsection{Examples under study}

Example 1: Take into consideration the following data from the 25 observations concerning the production of metal products suspected of variations in raw material iron content from five suppliers. Of each of the five suppliers, five ingots were randomly chosen. The below observations (Data 1) includes percentage by weight for the iron determination on each ingot.

Data 1:

\begin{tabular}{cccccc}
\hline Supplier & \multicolumn{5}{c}{ \% weight } \\
\hline 1 & 3.46 & 3.48 & 3.56 & 3.39 & 3.4 \\
2 & 3.59 & 3.46 & 3.42 & 3.49 & 3.5 \\
3 & 3.51 & 3.64 & 3.46 & 3.52 & 3.49 \\
4 & 3.38 & 3.4 & 3.37 & 3.46 & 3.39 \\
5 & 3.29 & 3.46 & 3.37 & 3.32 & 3.38 \\
\hline
\end{tabular}

Example 2: The study includes three battery bands. The life of the three brands is suspected (in weeks). The following findings (Data 2) are tested for five batteries of each brand. Test whether the lives of these battery brands are different at 5\% level of significance.

Data 2:

\begin{tabular}{cccccc}
\hline Brands & \multicolumn{5}{c}{ Life in Weeks } \\
\hline 1 & 100 & 96 & 92 & 96 & 92 \\
2 & 76 & 80 & 75 & 84 & 82 \\
3 & 108 & 100 & 96 & 98 & 100 \\
\hline
\end{tabular}

Example 3: Four catalysts are being examined which can influence the concentration of one component in a fluid blend of three components. The levels are earned as follows (Data 3). Test if the four catalysts have an equal $10 \%$ significant impact on the concentration.

Data 3:

\begin{tabular}{ccccc}
\hline Catalysts & \multicolumn{4}{c}{ Concentration Levels } \\
\hline 1 & 58.2 & 57.2 & 58.4 & 55.8 \\
2 & 56.3 & 54.5 & 57 & 55.3 \\
3 & 50.1 & 54.2 & 55.4 & 54.9 \\
4 & 52.9 & 49.9 & 50 & 51.7 \\
\hline
\end{tabular}


The table 4 below shows that NWED is the best model and shows a considerable linear link (correlation co-efficient) between sampled and population quantiles in raised examples, as demonstrated in the Q-Q plot.

Table 4. $r$ value

\begin{tabular}{ccc}
\hline Example & Normal Dist. & NWED \\
\hline 1 & 0.2067 & $\mathbf{0 . 9 5 5 4}$ \\
2 & 0.4149 & $\mathbf{0 . 8 7 6 8}$ \\
3 & 0.4447 & $\mathbf{0 . 8 8 1 1}$ \\
\hline
\end{tabular}
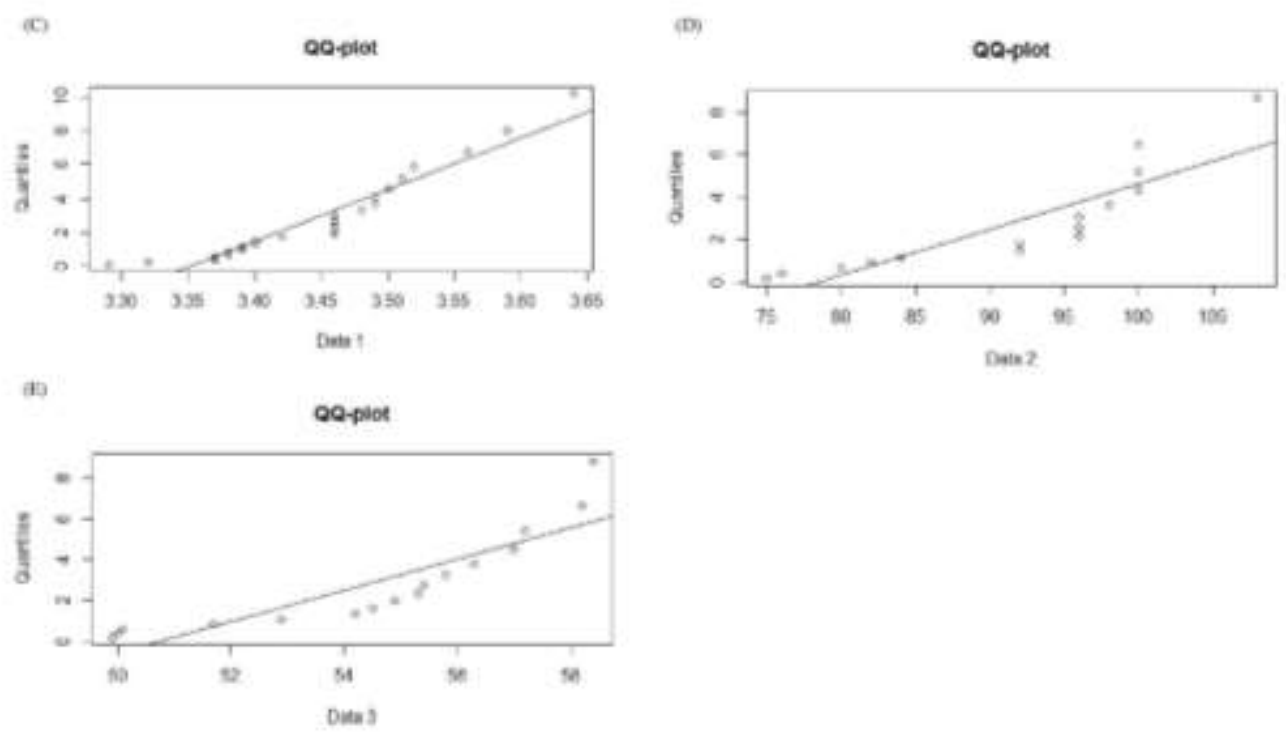

Figure 2 Graphs indicating QQ-plot for the data in the illustrated examples. (C): Example 1, (D): Example 2, (E): Example 3 
Table 5. NWED constants for Analysis of Means when $\alpha=0.01$

\begin{tabular}{|c|c|c|c|c|c|c|c|c|c|}
\hline$k / n$ & 2 & 3 & 4 & 5 & 6 & 7 & 8 & 9 & 10 \\
\hline \multirow{4}{*}{1} & & & 0.481 & 0.518 & 0.548 & 0.577 & 0.595 & 0.625 & 0.640 \\
\hline & 0.3758 & 0.4393 & 3 & 8 & 0 & 8 & 2 & 3 & 0 \\
\hline & 8.4359 & 6.9699 & 5.843 & 5.387 & 5.082 & 4.564 & 4.430 & 4.274 & 3.955 \\
\hline & & & 8 & 1 & 0 & 6 & 0 & 5 & 3 \\
\hline \multirow{4}{*}{2} & & & 0.444 & 0.487 & 0.522 & 0.550 & 0.562 & 0.600 & 0.610 \\
\hline & 0.3639 & 0.4130 & 0 & 7 & 1 & 8 & 8 & 3 & 3 \\
\hline & 9.5115 & 7.7933 & 6.359 & 5.857 & 5.730 & 4.863 & 4.849 & 4.528 & 4.201 \\
\hline & & & 9 & 3 & 2 & 6 & 9 & 0 & 0 \\
\hline \multirow{4}{*}{3} & & & 0.432 & 0.478 & 0.504 & 0.539 & 0.547 & 0.583 & 0.596 \\
\hline & 0.3567 & 0.4056 & 6 & 1 & 5 & 4 & 0 & 9 & 6 \\
\hline & 10.0172 & 8.2825 & 6.610 & 6.141 & 5.972 & 5.094 & 5.056 & 4.742 & 4.487 \\
\hline & & & 8 & 6 & 0 & 8 & 8 & 2 & 1 \\
\hline \multirow{4}{*}{4} & & & 0.427 & 0.470 & 0.491 & 0.522 & 0.537 & 0.577 & 0.584 \\
\hline & 0.3528 & 0.3998 & 0 & 8 & 2 & 2 & 2 & 9 & 0 \\
\hline & 10.1611 & 8.4739 & 6.761 & 6.224 & 6.037 & 5.321 & 5.137 & 4.868 & 4.556 \\
\hline & & & 5 & 4 & 7 & 0 & 2 & 2 & 7 \\
\hline \multirow{4}{*}{5} & & & 0.426 & 0.460 & 0.484 & 0.501 & 0.533 & 0.571 & 0.582 \\
\hline & 0.3502 & 0.3965 & 4 & 3 & 1 & 9 & 0 & 8 & 6 \\
\hline & 10.5592 & 8.5502 & 6.837 & 6.373 & 6.090 & 5.367 & 5.167 & 4.981 & 4.659 \\
\hline & & & 0 & 1 & 1 & 0 & 8 & 2 & 0 \\
\hline \multirow{4}{*}{6} & & & 0.425 & 0.458 & 0.471 & 0.491 & 0.528 & 0.558 & 0.575 \\
\hline & 0.3474 & 0.3957 & 1 & 1 & 8 & 8 & 3 & 2 & 9 \\
\hline & 10.7958 & 8.7261 & 6.857 & 6.515 & 6.222 & 5.577 & 5.253 & 5.058 & 4.772 \\
\hline & & & 8 & 0 & 1 & 9 & 0 & 3 & 3 \\
\hline \multirow{4}{*}{7} & & & 0.423 & 0.457 & 0.459 & 0.484 & 0.527 & 0.555 & 0.574 \\
\hline & 0.3473 & 0.3923 & 2 & 3 & 4 & 2 & 2 & 3 & 4 \\
\hline & 10.7964 & 9.0483 & 7.004 & 6.519 & 6.227 & 5.733 & 5.354 & 5.194 & 4.781 \\
\hline & & & 3 & 5 & 9 & 7 & 4 & 5 & 6 \\
\hline \multirow{4}{*}{8} & & & 0.415 & 0.456 & 0.458 & 0.484 & 0.526 & 0.548 & 0.574 \\
\hline & 0.3472 & 0.3921 & 1 & 3 & 7 & 0 & 1 & 2 & 3 \\
\hline & 10.7973 & 9.3994 & 7.348 & 6.704 & 6.271 & 5.820 & 5.394 & 5.278 & 4.966 \\
\hline & & & 7 & 5 & 7 & 0 & 3 & 4 & 9 \\
\hline \multirow{4}{*}{9} & & & 0.414 & 0.454 & 0.456 & 0.474 & 0.522 & 0.545 & 0.574 \\
\hline & 0.3449 & 0.3900 & 9 & 1 & 3 & 6 & 0 & 1 & 0 \\
\hline & 10.9862 & 9.4422 & 7.368 & 6.779 & 6.374 & 5.921 & 5.477 & 5.305 & 4.989 \\
\hline & & & 9 & 3 & 3 & 3 & 7 & 2 & 2 \\
\hline \multirow{4}{*}{10} & & & 0.414 & 0.454 & 0.456 & 0.474 & 0.522 & 0.545 & 0.574 \\
\hline & 0.3449 & 0.3900 & 9 & 1 & 3 & 6 & 0 & 1 & 0 \\
\hline & 10.9862 & 9.4422 & 7.368 & 6.779 & 6.374 & 5.921 & 5.477 & 5.305 & 4.989 \\
\hline & & & 9 & 3 & 3 & 3 & 7 & 2 & 2 \\
\hline \multirow{4}{*}{15} & & & 0.405 & 0.449 & 0.446 & 0.461 & 0.503 & 0.541 & 0.561 \\
\hline & 0.3399 & 0.3756 & 0 & 9 & 3 & 6 & 3 & 7 & 6 \\
\hline & 12.4806 & 10.0287 & 7.755 & 6.931 & 6.885 & 5.979 & 5.698 & 5.586 & 5.063 \\
\hline & & & 1 & 5 & 5 & 4 & 0 & 2 & 2 \\
\hline \multirow{4}{*}{20} & & & 0.395 & 0.445 & 0.436 & 0.459 & 0.501 & 0.534 & 0.558 \\
\hline & 0.3396 & 0.3756 & 9 & 1 & 5 & 9 & 6 & 3 & 5 \\
\hline & 13.3413 & 10.0287 & 7.844 & 6.960 & 7.011 & 6.243 & 5.920 & 5.913 & 5.097 \\
\hline & & & 9 & 4 & 7 & 4 & 7 & 1 & 3 \\
\hline \multirow{3}{*}{25} & & & 0.395 & 0.445 & 0.436 & 0.459 & 0.501 & 0.534 & 0.558 \\
\hline & $\begin{array}{l}0.3590 \\
133413\end{array}$ & $\begin{array}{l}0.3750 \\
10.0287\end{array}$ & 9 & 1 & 5 & 9 & 6 & 3 & 5 \\
\hline & & & 7.844 & 6.960 & 7.011 & 6.243 & 5.920 & 5.913 & 5.097 \\
\hline
\end{tabular}




\begin{tabular}{cccccccccc}
\hline & & & 9 & 4 & 7 & 4 & 7 & 1 & 3 \\
& & & 0.366 & 0.443 & 0.434 & 0.450 & 0.492 & 0.524 & 0.529 \\
30 & 0.3395 & 0.3638 & 8 & 5 & 1 & 4 & 8 & 7 & 3 \\
& 13.8106 & 10.4346 & 8.253 & 7.285 & 7.167 & 6.444 & 5.975 & 5.957 & 5.373 \\
& & & 5 & 1 & 8 & 1 & 1 & 5 & 1 \\
& & & 0.366 & 0.443 & 0.434 & 0.450 & 0.492 & 0.524 & 0.529 \\
35 & 0.3395 & 0.3638 & 8 & 5 & 1 & 4 & 8 & 7 & 3 \\
& 13.8106 & 10.4346 & 8.253 & 7.285 & 7.167 & 6.444 & 5.975 & 5.957 & 5.373 \\
& & & 5 & 1 & 8 & 1 & 1 & 5 & 1 \\
& & & 0.366 & 0.443 & 0.434 & 0.450 & 0.492 & 0.524 & 0.529 \\
40 & 0.3395 & 0.3638 & 8 & 5 & 1 & 4 & 8 & 7 & 3 \\
& 13.8106 & 10.4346 & 8.253 & 7.285 & 7.167 & 6.444 & 5.975 & 5.957 & 5.373 \\
& & & 5 & 1 & 8 & 1 & 1 & 5 & 1 \\
& & & 0.366 & 0.443 & 0.434 & 0.450 & 0.492 & 0.524 & 0.529 \\
45 & 0.3395 & 0.3638 & 8 & 5 & 1 & 4 & 8 & 7 & 3 \\
& 13.8106 & 10.4346 & 8.253 & 7.285 & 7.167 & 6.444 & 5.975 & 5.957 & 5.373 \\
& & & 5 & 1 & 8 & 1 & 1 & 5 & 1 \\
& & & 0.366 & 0.443 & 0.434 & 0.450 & 0.492 & 0.524 & 0.529 \\
50 & 0.3395 & 0.3638 & 8 & 5 & 1 & 4 & 8 & 7 & 3 \\
& 13.8106 & 10.4346 & 8.253 & 7.285 & 7.167 & 6.444 & 5.975 & 5.957 & 5.373 \\
& & & 5 & 1 & 8 & 1 & 1 & 5 & 1 \\
\hline
\end{tabular}

The constants mentioned in the above Table 5 are in particular used to illustrate the Example 1. For Data 1, the sample means are $\overline{x_{1}}=3.458, \overline{x_{2}}=3.492, \overline{x_{3}}=3.524, \overline{x_{4}}=3.400$ and $\overline{x_{5}}=3.364$. The overall mean or population mean is $\bar{x}=3.4476$. For stated $n=5$ and $k=5$, we use the percentiles in Eq. (5) to get $L^{*}=L \cdot \bar{x}$ (LDL) and $U^{*}=U \cdot \bar{x}$ (UDL) for $\alpha=0.0 .1$ (assumed). Those values (decision limits) are presented in Table 8. Here from Table 5, we can observe that, for $n=5$ and $k=5, L=0.4603$ and $U=6.3731$. These constants are useful in general, for specified $n$ and $k$. 
Table 6. NWED constants for Analysis of Means when $\alpha=0.05$

\begin{tabular}{|c|c|c|c|c|c|c|c|c|c|}
\hline$k / n$ & 2 & 3 & 4 & 5 & 6 & 7 & 8 & 9 & 10 \\
\hline \multirow{2}{*}{1} & 0.4458 & 0.5141 & 0.5715 & 0.5991 & 0.6293 & 0.6564 & 0.6739 & 0.7032 & 0.7135 \\
\hline & 5.7094 & 4.9388 & 4.3630 & 4.0340 & 3.9095 & 3.6288 & 3.4849 & 3.3865 & 3.2355 \\
\hline \multirow{2}{*}{2} & 0.4079 & 0.4723 & 0.5266 & 0.5641 & 0.5848 & 0.6201 & 0.4361 & 0.6605 & 0.6753 \\
\hline & 6.7877 & 5.7089 & 5.1072 & 4.5788 & 4.3758 & 4.0549 & 3.9040 & 3.7448 & 3.5865 \\
\hline \multirow{2}{*}{3} & 0.3929 & 0.4605 & 0.5105 & 0.5417 & 0.5722 & 0.6050 & 0.6218 & 0.6413 & 0.6564 \\
\hline & 7.3963 & 6.2438 & 5.4008 & 4.9023 & 4.7266 & 4.2877 & 4.1333 & 4.0069 & 3.7727 \\
\hline \multirow{2}{*}{4} & 0.3848 & 0.4471 & 0.4965 & 0.5299 & 0.5562 & 0.5887 & 0.6028 & 0.6325 & 0.6477 \\
\hline & 8.2010 & 6.5604 & 5.7067 & 5.2001 & 4.8805 & 4.4533 & 4.3297 & 4.1791 & 3.8887 \\
\hline \multirow{2}{*}{5} & 0.3766 & 0.4397 & 0.4827 & 0.5205 & 0.5511 & 0.5815 & 0.5972 & 0.6260 & 0.6407 \\
\hline & 8.4269 & 6.9514 & 5.8377 & 5.3867 & 5.0590 & 4.5643 & 4.4046 & 4.2640 & 3.9496 \\
\hline \multirow{2}{*}{6} & 0.3715 & 0.4315 & 0.4754 & 0.5137 & 0.5413 & 0.5675 & 0.5850 & 0.6207 & 0.6354 \\
\hline & 8.7205 & 7.0962 & 5.9559 & 5.4669 & 5.1890 & 4.6358 & 4.5386 & 4.3727 & 4.0430 \\
\hline \multirow{2}{*}{7} & 0.3696 & 0.4246 & 0.4664 & 0.5092 & 0.5371 & 0.5616 & 0.5790 & 0.6139 & 0.6313 \\
\hline & 8.8756 & 7.3175 & 6.1244 & 5.6066 & 5.3184 & 4.7158 & 4.5894 & 4.4027 & 4.0873 \\
\hline \multirow{2}{*}{8} & 0.3675 & 0.4213 & 0.4595 & 0.4998 & 0.5306 & 0.5545 & 0.5713 & 0.6103 & 0.6255 \\
\hline & 9.2301 & 7.5617 & 6.2676 & 5.7637 & 5.3723 & 4.7724 & 4.6715 & 4.4739 & 4.1779 \\
\hline \multirow{2}{*}{9} & 0.3664 & 0.4174 & 0.4555 & 0.4917 & 0.5241 & 0.5532 & 0.5670 & 0.6037 & 0.6227 \\
\hline & 9.2951 & 7.6507 & 6.3244 & 5.8271 & 5.4936 & 4.8445 & 4.6969 & 4.5019 & 4.1922 \\
\hline \multirow{2}{*}{10} & 0.3639 & 0.4130 & 0.4440 & 0.4877 & 0.5221 & 0.5508 & 0.5628 & 0.6003 & 0.6103 \\
\hline & 9.5115 & 7.7933 & 6.3599 & 5.8573 & 5.7302 & 4.8636 & 4.8499 & 4.5280 & 4.2010 \\
\hline \multirow{2}{*}{15} & 0.3579 & 0.4058 & 0.4345 & 0.4800 & 0.5055 & 0.5399 & 0.5477 & 0.5892 & 0.5990 \\
\hline & 9.9812 & 8.2613 & 6.6065 & 6.0942 & 5.9519 & 5.0645 & 5.0405 & 4.7397 & 4.4420 \\
\hline \multirow{2}{*}{20} & 0.3528 & 0.3998 & 0.6270 & 0.4708 & 0.4912 & 0.5222 & 0.5372 & 0.5779 & 0.5840 \\
\hline & 10.1611 & 8.4739 & 6.7615 & 6.2244 & 6.0377 & 5.3210 & 5.1372 & 4.8682 & 4.5567 \\
\hline \multirow{2}{*}{25} & 0.3502 & 0.3965 & 0.4264 & 0.4603 & 0.4841 & 0.5019 & 0.5330 & 0.5718 & 0.5826 \\
\hline & 10.5592 & 8.5502 & 6.8370 & 6.3731 & 6.0901 & 5.3670 & 5.1678 & 4.9812 & 4.6590 \\
\hline \multirow{2}{*}{30} & 0.3474 & 0.3957 & 0.4251 & 0.4581 & 0.4718 & 0.4918 & 0.5283 & 0.5582 & 0.5759 \\
\hline & 10.7958 & 8.7261 & 6.8578 & 6.5150 & 6.2221 & 5.5779 & 5.2530 & 5.0583 & 4.7723 \\
\hline \multirow{2}{*}{35} & 0.3473 & 0.3923 & 0.4232 & 0.4573 & 0.4596 & 0.4842 & 0.5272 & 0.5553 & 0.5744 \\
\hline & 10.7964 & 9.0483 & 7.0043 & 6.5195 & 6.2279 & 5.7337 & 5.3544 & 5.1945 & 4.7816 \\
\hline \multirow{2}{*}{40} & 0.3472 & 0.3921 & 0.4151 & 0.4563 & 0.4587 & 0.4840 & 0.5261 & 0.5482 & 0.5743 \\
\hline & 10.7973 & 9.3994 & 7.3487 & 6.7045 & 6.2717 & 5.8200 & 5.3943 & 5.2784 & 4.9669 \\
\hline \multirow{2}{*}{45} & 0.3449 & 0.3900 & 0.4149 & 0.4541 & 0.4563 & 0.4746 & 0.5220 & 0.4551 & 0.5740 \\
\hline & 10.9862 & 9.4422 & 7.3689 & 6.7793 & 6.3743 & 5.5213 & 5.4777 & 5.3052 & 4.9892 \\
\hline \multirow{2}{*}{50} & 0.3449 & 0.3900 & 0.4149 & 0.4541 & 0.4563 & 0.4746 & 0.5220 & 0.4551 & 0.5740 \\
\hline & 10.9862 & 9.4422 & 7.3689 & 6.7793 & 6.3743 & 5.5213 & 5.4777 & 5.3052 & 4.9892 \\
\hline
\end{tabular}

In specifically for Example 2 the constants specified in the above-described Table 6 are employed. For Data 2, the sample means are $\overline{x_{1}}=95.2, \overline{x_{2}}=79.4$, and $\overline{x_{3}}=100.4$. The overall mean or population mean is $\bar{x}=91.6667$. For stated $n=5$ and $k=3$, and for $\alpha=0.05$, from Table 6 we can find $L=0.5417$ and $U=4.9023$.

The same technique is used and the calculated values (decision limits) are presented in Table 8. In general, for stated $n$ and $k$, these constants are helpful. 
Table 7 NWED constants for Analysis of Means when $\alpha=0.10$

\begin{tabular}{|c|c|c|c|c|c|c|c|c|c|}
\hline$k / n$ & 2 & 3 & 4 & 5 & 6 & 7 & 8 & 9 & 10 \\
\hline \multirow{2}{*}{1} & 0.4987 & 0.5664 & 0.6202 & 0.6516 & 0.6811 & 0.7027 & 0.7245 & 0.7549 & 0.7693 \\
\hline & 4.6020 & 4.1144 & 3.6551 & 3.5009 & 3.3762 & 3.1799 & 3.0881 & 2.9910 & 2.8649 \\
\hline \multirow{2}{*}{2} & 0.4466 & 0.5156 & 0.5740 & 0.6027 & 0.6307 & 0.6580 & 0.6754 & 0.7040 & 0.7153 \\
\hline & 5.6535 & 4.9046 & 4.3465 & 4.0223 & 3.8997 & 3.6140 & 3.4753 & 3.3791 & 3.2288 \\
\hline \multirow{2}{*}{3} & 0.4232 & 0.4913 & 0.5491 & 0.5788 & 0.6059 & 0.6339 & 0.6541 & 0.6746 & 0.6924 \\
\hline & 6.3360 & 5.3818 & 4.7362 & 4.3044 & 4.1303 & 3.8526 & 3.7258 & 3.5863 & 3.4126 \\
\hline \multirow{2}{*}{4} & 0.4087 & 0.4736 & 0.5275 & 0.5651 & 0.5860 & 0.6207 & 0.6376 & 0.6613 & 0.6768 \\
\hline & 6.7391 & 5.6888 & 5.0604 & 4.5437 & 4.3683 & 4.0484 & 3.8818 & 3.7344 & 3.5788 \\
\hline \multirow{2}{*}{5} & 0.4017 & 0.4651 & 0.5169 & 0.5538 & 0.5783 & 0.6137 & 0.6303 & 0.6519 & 0.6639 \\
\hline & 7.0563 & 5.9478 & 5.2376 & 4.7470 & 4.5269 & 4.1721 & 4.0153 & 3.8608 & 3.7021 \\
\hline \multirow{2}{*}{6} & 0.3942 & 0.4616 & 0.5112 & 0.5428 & 0.5731 & 0.6066 & 0.6257 & 0.6434 & 0.6567 \\
\hline & 7.3281 & 6.2211 & 5.3846 & 4.8809 & 4.7038 & 4.2772 & 4.1176 & 3.9849 & 3.7604 \\
\hline \multirow{2}{*}{7} & 0.3891 & 0.4536 & 0.5007 & 0.5329 & 0.5650 & 0.5956 & 0.6170 & 0.6382 & 0.6528 \\
\hline & 7.8144 & 6.3411 & 5.4845 & 5.0271 & 4.7970 & 4.3640 & 4.2659 & 4.1098 & 3.8256 \\
\hline \multirow{2}{*}{8} & 0.3855 & 0.4480 & 0.4969 & 0.5303 & 0.5575 & 0.5901 & 0.6031 & 0.6339 & 0.6489 \\
\hline & 8.1552 & 6.5107 & 5.6939 & 5.1790 & 4.8684 & 4.4314 & 4.3253 & 4.1583 & 3.8837 \\
\hline \multirow{2}{*}{9} & 0.3809 & 0.4430 & 0.4919 & 0.5251 & 0.5533 & 0.5858 & 0.6009 & 0.6298 & 0.6467 \\
\hline & 8.3170 & 6.6693 & 5.7913 & 5.2670 & 4.9351 & 4.4924 & 4.3495 & 4.2272 & 3.9189 \\
\hline \multirow{2}{*}{10} & 0.3771 & 0.4400 & 0.4837 & 0.5207 & 0.5512 & 0.5820 & 0.5976 & 0.6269 & 0.6410 \\
\hline & 8.4220 & 6.9182 & 5.8356 & 5.3396 & 5.0556 & 4.5456 & 4.4039 & 4.2557 & 3.9462 \\
\hline \multirow{2}{*}{15} & 0.3694 & 0.4245 & 0.4650 & 0.5036 & 0.5343 & 0.5592 & 0.5746 & 0.6130 & 0.6298 \\
\hline & 8.9986 & 7.5022 & 6.1891 & 5.6447 & 5.3532 & 4.7393 & 4.6198 & 4.4367 & 4.1214 \\
\hline \multirow{2}{*}{20} & 0.3657 & 0.4152 & 0.4453 & 0.4883 & 0.5231 & 0.5519 & 0.5637 & 0.6007 & 0.6159 \\
\hline & 9.4710 & 7.7531 & 6.3450 & 5.8334 & 5.6720 & 4.8620 & 4.7882 & 4.5202 & 4.1993 \\
\hline \multirow{2}{*}{25} & 0.3616 & 0.4092 & 0.4416 & 0.4845 & 0.5127 & 0.5444 & 0.5564 & 0.5930 & 0.6042 \\
\hline & 9.6045 & 7.9203 & 6.4883 & 5.9039 & 5.8046 & 4.9569 & 4.9291 & 4.6799 & 4.2671 \\
\hline \multirow{2}{*}{30} & 0.3579 & 0.4058 & 0.4345 & 0.4800 & 0.5055 & 0.5399 & 0.5477 & 0.5892 & 0.5990 \\
\hline & 9.9812 & 8.2613 & 6.6065 & 6.0942 & 5.9519 & 5.0645 & 5.0405 & 4.7397 & 4.4420 \\
\hline \multirow{2}{*}{35} & 0.3558 & 0.4010 & 0.4309 & 0.4773 & 0.4998 & 0.5388 & 0.5423 & 0.5830 & 0.5956 \\
\hline & 10.0213 & 8.3186 & 6.6456 & 6.1472 & 5.9777 & 5.2318 & 5.0691 & 4.7549 & 4.4924 \\
\hline \multirow{2}{*}{40} & 0.3529 & 0.4002 & 0.4279 & 0.4713 & 0.4937 & 0.5266 & 0.5414 & 0.5785 & 0.5909 \\
\hline & 10.1224 & 8.4559 & 6.7542 & 6.2198 & 6.0049 & 5.2979 & 5.1336 & 4.8645 & 4.5500 \\
\hline \multirow{2}{*}{45} & 0.3510 & 0.3986 & 0.4264 & 0.4632 & 0.4857 & 0.5073 & 0.5361 & 0.5741 & 0.5827 \\
\hline & 10.1920 & 8.5110 & 6.8009 & 6.2496 & 6.0673 & 5.3539 & 5.1514 & 4.8718 & 4.6157 \\
\hline \multirow{2}{*}{50} & 0.3502 & 0.3965 & 0.4264 & 0.4603 & 0.4841 & 0.5019 & 0.5330 & 0.5718 & 0.5826 \\
\hline & 10.5592 & 8.5502 & 6.8370 & 6.3731 & 6.0901 & 5.3670 & 5.1678 & 4.9812 & 4.6590 \\
\hline
\end{tabular}

In specifically for Example 3 the constants stated in the above-described Table 7 are applied. For Data 3, the sample means are $\overline{x_{1}}=57.35, \overline{x_{2}}=55.775, \overline{x_{3}}=53.65$, and $\overline{x_{4}}=51.125$. The overall mean or population mean is $\bar{x}=54.475$. For stated $n=4$ and $k=4$, and $\alpha=0.10$, we may find from Table 7, $L=0.5275$ and $U=5.0604$. Using the same technique, Table 8 provides and shows derived values (decision limits). These constants are usually beneficial for specified $n$ and $k$. 
We have determined and submitted Table 8, the decision limits (DL) for the Normal Population and the NWED Population utilizing these findings in data as a single sample.

Table 8. Comparison between Normal Distribution and NWED

\begin{tabular}{|c|c|c|c|c|c|c|}
\hline \multirow[b]{2}{*}{$\begin{array}{c}\text { Example } \\
\text { No. } \\
(n, k, \alpha)\end{array}$} & \multicolumn{3}{|c|}{ Normal Dist. } & \multicolumn{3}{|c|}{ NWED } \\
\hline & $\begin{array}{l}\text { Decision } \\
\text { Limits } \\
\text { [LDL, } \\
\text { UDL] }\end{array}$ & $\begin{array}{l}\text { Count } I \\
\text { (Within } \\
\text { Limits) }\end{array}$ & $\begin{array}{l}\text { Probability } \\
(p=i / k)\end{array}$ & $\begin{array}{c}\text { Decision } \\
\text { Limits } \\
\text { [LDL, } \\
\text { UDL] }\end{array}$ & $\begin{array}{l}\text { Count } i \\
\text { (Within } \\
\text { Limits) }\end{array}$ & $\begin{array}{l}\text { Probability } \\
(p=i / k)\end{array}$ \\
\hline $\begin{array}{c}1 \\
(5,5, \\
0.01)\end{array}$ & $\begin{array}{l}{[3.517,} \\
3.879]\end{array}$ & 3 & 0.6 & $\begin{array}{l}{[1.587,} \\
21.972]\end{array}$ & 5 & 1 \\
\hline $\begin{array}{c}2 \\
(5,3, \\
0.05)\end{array}$ & $\begin{array}{l}{[87.82,} \\
95.52]\end{array}$ & 2 & 0.7 & $\begin{array}{l}\text { [49.656, } \\
449.378]\end{array}$ & 3 & 1 \\
\hline $\begin{array}{c}3 \\
(4,4, \\
0.10)\end{array}$ & $\begin{array}{l}{[26.14,} \\
82.84]\end{array}$ & 2 & 0.5 & $\begin{array}{l}{[28.735,} \\
275.665]\end{array}$ & 4 & 1 \\
\hline
\end{tabular}

$n$ : Size of Subgroup, $k$ : No. of Subgroups, $\alpha$ : level of significance, LDL: Lower Decision Limit, UDL: Upper Decision Limit.

\section{Conclusion}

According to the decision limits using Normal distribution or the Shewart control limits and ANOM tables of Ott.E.R [7], the number of homogenous mean is 3,2, and 2 for each data set, and those who are not homogeneous are 2, 1, and 2 respectively. When the ANOM tables of NWED are utilized, the number of homogeneous means for the same data sets is 5,3 , and 4 , with no deviations from the homogeneity. This indicates that, when the normal distribution model has been applied, certain means have been homogenized, and others have deviated. This decision is valid, even if the data corresponds to the Normal distribution. In comparison, NWED is a better model that has already been displayed on a Q-Q plot that shows that each data set with Normal and NWED has a Q-Q correlation coefficient independently. As a result, we concluded that the decision method of the Normal distribution will be correlated with more error. Henceforth, using proposed NWED model is a better option rather than the usual, to achieve homogeneity for ANOM method in some cases. 


\section{References}

[1] Bakir.S.T., Means using ranks for randomised complete block designs. Communications in Statistics Simulation and Computation. 1994; 23:547-568.

[2] Bernard.A.J., and Wludyka.P.S. Robust I-sample Analysis of means type randomization tests for variances Journal of Statistical Computation and Simulation. 2001; 69:57-88.

[3] Farnum.N.R. Analysis of Means Tables using mathematical processors. Quality Engineering. 2004; 16:399-405.

[4] Guirguis.G.H., and Tobias.R.D. On the computation of the distribution for the analysis of means. Communications in Statistics- Simulation and Computation. 2004; 16:861-887.

[5] Montgomery.D.C. Design and Analysis of Experiments. Fifth edition, John Wiley and Sons, New York.

[6] Nelson.P.R., and Dudewicz.E.J. Exact Analysis of Means with Unequal Variances. Technometrics. 2002; 44:152-160.

[7] Ott.E.R. Analysis of Means- A graphical procedure. Industrial Quality Control. 1967; 24:101-109.

[8] Ramig.P.F. Applications of Analysis of Means. Journal of Quality Technology. 1983; 15:19-25.

[9] Rao.C.V. Analysis of Means - A review. Journal of Quality Technology. 2005; 37:308-315.

[10] Rao.C.V., and Prankumar.M. ANOM-type Graphical Methods for testing the Equality of Several Correlation Coefficients. Gujarat Statistical Review. 2002; 29:47-56.

[11] Srinivasa Rao.B., Pratapa Reddy.J., and Rosaiah.K. Extreme value charts and ANOM based on inverse Rayleigh distribution. Pakistan Journal of Statistic \& Operations Research. 2012; 8(4):759-766.

[12] Srinivasa Rao.B., and Kantam.R.R.L. Extreme value charts and Analysis of means based on half logistic distribution. International Journal of Quality, Reliability and Management. 2012; 29(5):501-511.

[13] Srinivasa Rao.B., and Sricharani.P. Extreme value charts and Analysis of means based on Dagum distribution. International Journal of Statisytics \& Applied maythematics. 2018; 3(2):351-354.

[14] Srinivasa Rao.B., Pratapa Reddy.J., and Sarath Babu.G. Extreme value charts and ANOM based on log-logistic distribution. Modern Applied Statistical Methods. 2012; 493-505. 